\title{
Testing General Relativity with Pulsar Timing Arrays
}

\author{
Sydney J. Chamberlin and Xavier Siemens \\ Department of Physics, University of Wisconsin-Milwaukee, Milwaukee, WI
}

\begin{abstract}
Pulsar timing arrays are a promising tool for probing the universe through gravitational radiation. A variety of astrophysical and cosmological sources are expected to contribute to a stochastic background of gravitational waves (GWs) in the pulsar timing array (PTA) frequency band. Direct detection of GWs will provide a new mechanism to test General Relativity and requires the development of robust statistical detection strategies. Here, we investigate the overlap reduction function, a term present in the optimal detection statistic, for GWs in various metric theories of gravity. We show that PTA sensitivity increases for non-transverse gravitational waves when pulsar pairs have small angular separations in the sky.
\end{abstract}

\section{Introduction}

Astrophysical and cosmological objects such as coalescing supermassive black hole binaries, asymmetrical rotating neutron stars and supernovae, and other exotic sources such as cosmic strings are expected to produce a stochastic background of gravitational radiation in the universe. Mathematically, such gravitational waves (GWs) can manifest in up to six different polarization modes, shown in Figure 1. Einstein's theory of General Relativity (GR), however, restricts the six possible modes to just two. In the past half-century, a number of viable alternative theories of gravity have emerged. Many of these theories satisfy weak-field, slow motion tests of gravity such as the bending of light around massive objects and the precession of the perihelion of Mercury, but differ from GR in the predictions they make regarding GWs. A direct detection of GWs thus presents a unique opportunity to test GR.

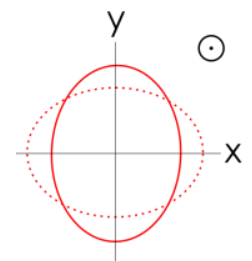

(a) plus mode

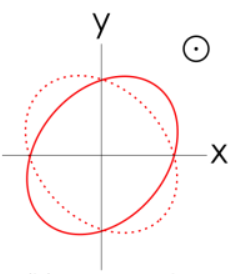

(b) cross mode

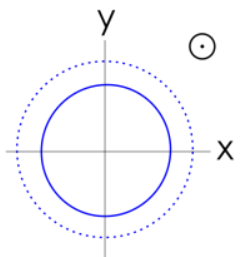

(c) breathing mode

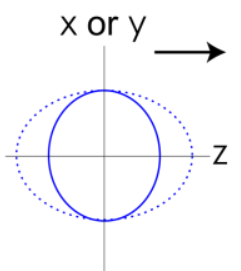

(d) longitudinal mode

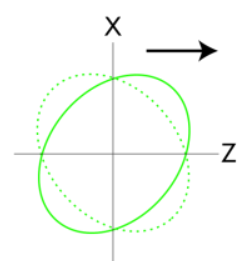

(e) vector-x mode

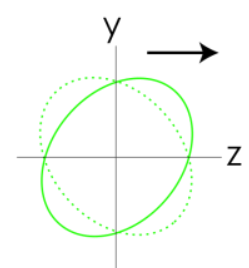

(f) vector-y mode

Figure 1: In a general metric theory of gravity, as many as six GW polarization modes are possible. Note that Fig. 1(a)-(c) represent transverse wave propagation, with the plus and cross modes corresponding to GR; Fig. 1 (d)-(f) represent non-transverse wave propagation. Fig. 1(a) and 1(b) correspond to the plus/cross modes of GR.

Acknowledgments: We thank the Wisconsin Space Grant Consortium and the National Science Foundation for their support in this project. We would also like to thank our collaborators in the North American Nanohertz Observatory for Gravitational Waves (NANOGrav). 
A number of large-scale GW detection efforts are currently underway world-wide, with current focus primarily given to ground-based laser interferometric detectors, sensitive in the $10-10^{3}$ $\mathrm{Hz}$ range (Abramovici et al. 1992), and pulsar timing arrays, sensitive in the $10^{-9}-10^{-7} \mathrm{~Hz}$ range (Hobbs et al. 2010). The work presented in this project involves detection efforts using pulsar timing arrays (PTAs).

\section{Detecting Gravitational Waves with Pulsar Timing Arrays}

The radio signals emitted by many pulsars are stable enough to serve as clocks. A GW, if present between the Earth and the pulsar, will induce a redshift in the pulsar's signal. This redshift will cause the pulse to arrive either early or late at the Earth. For a single pulsar, this information is not very useful since a number of other physical factors could account for the pulse's delay. With an entire array of pulsars, however, the GW will induce redshifts in the pulsars' signals in a correlated way. PTAs are used in the search for GWs by seeking out the correct crosscorrelations between data from different pulsar pairs.

The primary focus of this work is the development of statistical data analysis tools that are necessary to extract the tiny GW signal from noise in data. The so-called optimal detection statistic is found by maximizing the expected signal-to-noise ratio (Anholm et al. 2009), and involves calculating the overlap reduction function $\Gamma(\mathrm{f})$, a geometrical quantity that appears in the expression for cross-correlation. The overlap reduction function is related to the loss in sensitivity due to pulsars not being co- located or co-aligned, and characterizes the sensitivity of the detector to a $\mathrm{GW}$ given its polarization mode.

\section{Overlap Reduction Functions}

Overlap reduction functions were calculated for all six possible $\mathrm{GW}$ polarization modes by integrating the proper geometric expressions. To obtain meaningful analytic results, each pulsar pair was considered equidistant $(\mathrm{L} 1=\mathrm{L} 2=\mathrm{L}$, where $\mathrm{L}$ is the distance to the pulsar from the Earth) and several different pulsar separation angles $\xi$ were chosen. The results are functions of $\mathrm{fL}$, where $\mathrm{f}$ is the $\mathrm{GW}$ frequency in $\mathrm{Hz}$, and $\mathrm{L}$ is the geometrized distance to the pulsars; these are shown in Figure 2a-d. Here we point out that we have excluded plots for the cross and vector-x modes, as they can be described by the plus and vector-y mode without loss of generality.

Also plotted in Figure 2 are the curves obtained by excluding the pulsar term (this is the term containing all the frequency dependence of the $\mathrm{GW}$ and distance to the pulsar) in the integration of the overlap reduction function. This is a technique that is often used in the literature to simplify calculations for GR, and has been justified by examining the agreement between the dashed and solid lines in Figures $2 \mathrm{a}$ and $2 \mathrm{~b}$. We found that for non-transverse GWs, the overlap reduction functions retain frequency dependence well into the range of fL that is relevant to pulsar timing experiments, meaning the pulsar term cannot be excluded for these polarization modes. 
One can also see by studying Figure 2 that for non-transverse GWs, the values obtained by $\Gamma(\mathrm{fL})$ are much larger than those of the transverse GWs, especially for pulsar pairs with smaller angular separations. This increase in $\Gamma(\mathrm{fL})$ may be interpreted physically as an increase in sensitivity, and means that pulsar timing arrays should be much more sensitive to vector and scalar longitudinal polarized GWs than to the ordinary GWs of GR.

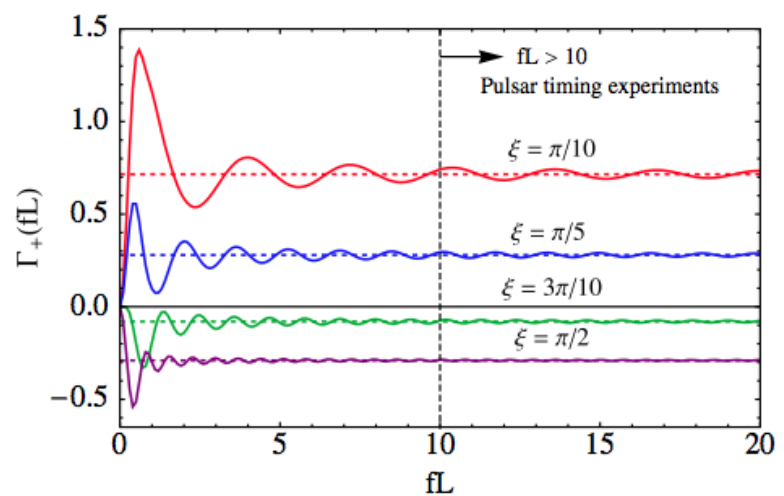

(a)

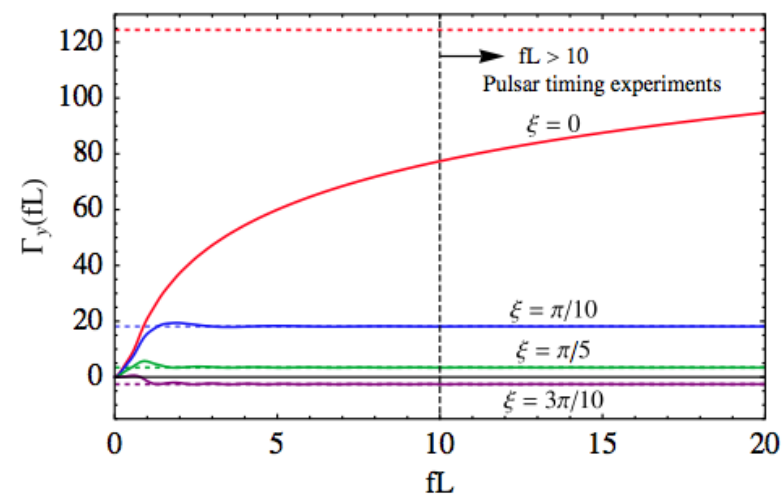

(c)

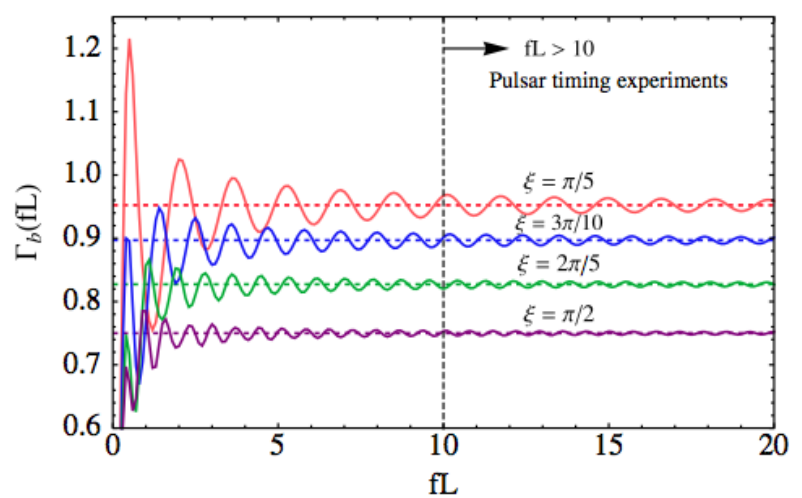

(b)

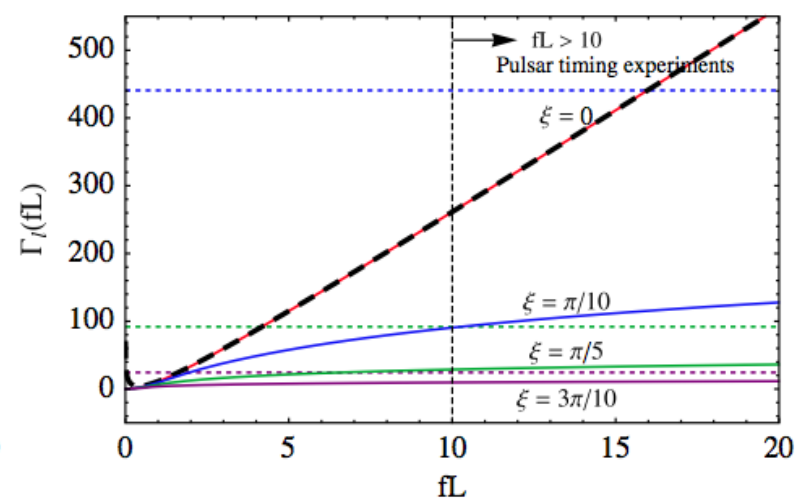

(d)

Figure 2: $\Gamma(\mathrm{fL})$ with (solid curves) and without (horizontal dashed lines) the pulsar term for the various polarization modes: plus (a), breathing (b), vector-y (c), and longitudinal (d). In the latter two modes, smaller pulsar separation angles $\xi$ are characterized by retained frequency dependence in $\Gamma(\mathrm{fL})$ in the range of frequencies relevant to pulsar timing experiments. Nearly all the non-transverse curves eventually converge, but at rather high values of $\Gamma(\mathrm{fL})$ relative to the transverse modes, indicating increased sensitivity to GWs with these polarizations.

The physical origin of this effect and calculations of the overlap reduction functions for actual current pulsar timing array data are described more fully in Chamberlin and Siemens (2012).

\section{Conclusion}

It is possible that a direct detection of GWs will be made in the next decade using a pulsar timing array. Such detections will provide new testing grounds for GR. To assist in the development of robust statistical detection strategies, we have analyzed overlap reduction functions for six possible GW polarization modes in a metric theory of gravity. We have found that PTAs should be much more sensitive to GWs with vector and scalar longitudinal 
polarizations that are present in some alternative theories of gravity than they are to the polarization modes of GR for pulsar pairs that have small angular separation.

\section{Works Cited}
A. Abramovici, W. E. Althouse, R. W. Drever, Y. Gursel, S. Kawamura, F. J. Raab, D. Shoemaker, L. Sievers, R. E. Spero, K. S. Thorne, R. E. Vogt, R. Weiss, S. E. Whitcomb, and M. E. Zucker, Science 256, 325 (1992).
M. Anholm, S. Ballmer, J. D. E. Creighton, L. R. Price, and X. Siemens, Physical Review D 79, 084030 (2009).

S. J. Chamberlin and X. Siemens, Physical Review D 85, 082001 (2012).

G. Hobbs et al., Classical Quantum Gravity 27, 084013 (2010). 